\title{
EVALUACIÓN DE LA SOSTENIBILIDAD DE LAS ALMAZARAS EN ANDALUCÍA (ESPAÑA): UN ESTUDIO BASADO EN INDICADORES COMPUESTOS
}

\author{
Victoria Vicario-Modroño, Rosa Gallardo-Cobos*, Pedro Sánchez-Zamora \\ Departamento de Economía Agraria, ETSIAM, Universidad de Córdoba (Córdoba, td2vimav@uco.es)
}

\begin{abstract}
Resumen
En los últimos tiempos, la necesidad de contar con sistemas alimentarios que además de ser económicamente viables y socialmente equitativos, dispongan de procesos de producción respetuosos con el medio ambiente, ha convertido la elaboración sostenible del aceite de oliva en una de las principales preocupaciones y prioridades del sector. En este contexto, la evaluación del desempeño económico, social y ambiental de las empresas oleícolas y el diseño de alternativas de gestión sostenible se han convertido en actividades fundamentales. Así, el objetivo principal de esta comunicación es medir la sostenibilidad de una muestra representativa de almazaras ubicadas en Andalucía (España), principal región productora de aceite de oliva a nivel internacional, e identificar cuáles son los factores determinantes que influyen sobre ella. Los resultados muestran que factores como la dimensión y el tamaño empresarial, la apuesta por la calidad, y la formación y profesionalización de los gestores son elementos clave para el desarrollo sostenible de las almazaras. Estos resultados pueden ser de utilidad para los responsables de la empresa en el diseño de estrategias encaminadas a mejorar su sostenibilidad.
\end{abstract}

Palabras clave: Sostenibilidad; Almazaras; Aceite de oliva; Indicadores; Andalucía 1. Introducción

En los últimos años ha habido un incremento del interés de la comunidad internacional por avanzar hacia sistemas alimentarios sostenibles caracterizados por proporcionar seguridad alimentaria y nutrición para la sociedad actual de manera que no se comprometan las bases de las generaciones futuras (Bellantuono et al., 2018). Para ello, la evaluación del desempeño ambiental, social y económico de los sistemas alimentarios y el diseño de alternativas de gestión sostenibles son, probablemente, el verdadero desafío de las próximas décadas (Notarnicola et al., 2017).

En la Cuenca Mediterránea, uno de los principales sistemas alimentarios es el que se conforma en torno al cultivo del olivar y la producción de aceite de oliva. España es el principal productor mundial de este tipo de aceite y Andalucía es la región oleícola más importante, cuya producción representa más del $80 \%$ del aceite nacional, el 65\% de la producción europea y más del 45\% del aceite mundial (Junta de Andalucía, 2020). Se trata de un sector en evolución en el que en las últimas décadas se han producido importantes transformaciones, tanto en la fase agraria (aumentos de la superficie, incorporación de nuevas variedades, tendencia hacia sistemas más competitivos y prácticas más intensivas, etc.) como en la fase de transformación y elaboración del aceite de oliva. Esta circunstancia ha tenido importantes implicaciones económicas y sociales, pero también medioambientales (contaminación acústica y emisiones de GEI, vertidos y generación de residuos, etc.) (Roig et al., 2006).

Para responder a esta situación, la elaboración sostenible de aceite de oliva se ha convertido en una de las principales preocupaciones y prioridades del sector. En este sentido, además de la respuesta proporcionada en la fase de producción agraria a través de prácticas como la intensificación sostenible, la agricultura ecológica o la integrada, también destaca la llevada a cabo en la fase industrial con innovaciones tales como la implantación del sistema de dos fases en la extracción del aceite de oliva o la reutilización de subproductos. Se trata de algunas innovaciones que han sido incorporadas por las almazaras y que les permite avanzar hacia algunos aspectos del desarrollo sostenible.

Sin embargo, para identificar y aplicar las opciones más sostenibles en cualquier ámbito del sector agroalimentario es necesario realizar una evaluación sobre la forma en la que se lleva a cabo la elaboración del alimento (Ahmad et al., 2019). Para ello, los indicadores de desarrollo sostenible están reconocidos como herramientas útiles para evaluar y anticipar el rendimiento y las tendencias de la producción, proporcionando información de alerta temprana y ayudando a la toma de decisiones de cualquier sistema de gestión (Salvado et al., 2015).

En el sector oleícola son numerosos los estudios que han analizado la sostenibilidad, principalmente desde un punto de vista medioambiental. Sin embargo, son escasos los trabajos que han analizado conjuntamente las tres dimensiones de la sostenibilidad en la fase industrial de este sector. En este contexto, el objetivo principal de la comunicación es medir la sostenibilidad de una muestra representativa de las almazaras ubicadas en Andalucía (España), e identificar cuáles son los factores determinantes que influyen sobre ella, tanto a nivel global como en cada una de sus dimensiones.

\section{Metodología}

La muestra seleccionada para realizar el análisis de sostenibilidad la conforman 81 almazaras cooperativas 
oleícolas de Andalucía. El análisis llevado a cabo se enmarca en la línea de trabajos basados en la construcción de indicadores e índices de sostenibilidad. En este sentido, en el Cuadro 1 se muestran las cuatro fases de la metodología empleada para la consecución del objetivo principal y los métodos estadísticos involucrados en cada una de ellas.

Cuadro 1. Metodología de la medición de la sostenibilidad

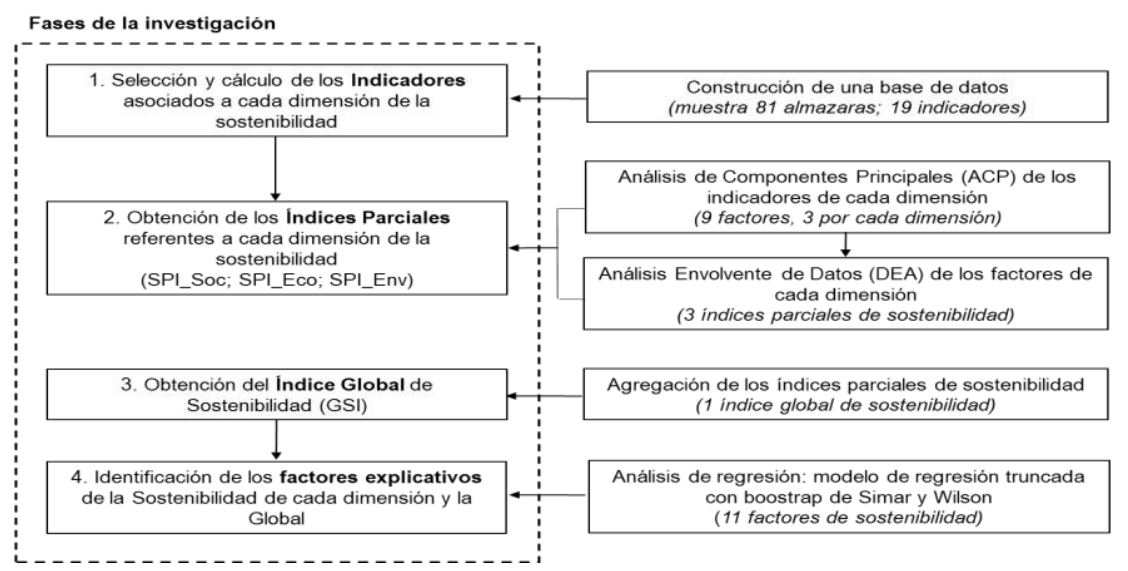

En la fase 1 se han seleccionado y calculado los indicadores asociados a cada una de las dimensiones de la sostenibilidad (ver Cuadro 2). Para ello se han seguido las orientaciones y esquemas descritos en la literatura especializada.

Cuadro 2. Indicadores de sostenibilidad

\begin{tabular}{|c|c|c|c|c|}
\hline Dimensión & Tema & Criterio & Indicador & Definición \\
\hline \multirow{7}{*}{ Social } & \multirow{4}{*}{ Empleo } & \multirow{2}{*}{$\begin{array}{l}\text { Plantilla total por tipo de } \\
\text { empleo }\end{array}$} & $\begin{array}{c}\text { Personal fijo } \\
\text { (PERS F) }\end{array}$ & $\begin{array}{l}\text { Porcentaje de trabajadores fijos con respecto al } \\
\text { total de trabajadores }(\%)\end{array}$ \\
\hline & & & $\begin{array}{c}\text { Personal especializado } \\
\text { (PERS_ESP) }\end{array}$ & $\begin{array}{l}\text { Porcentaje de personal especializado con } \\
\text { respecto al total de trabajadores }(\%)\end{array}$ \\
\hline & & $\begin{array}{l}\text { No discriminación e } \\
\text { igualdad de oportunidades. } \\
\text { Derechos laborales }\end{array}$ & $\begin{array}{l}\text { Igualdad de género } \\
\text { (MUI) }\end{array}$ & $\begin{array}{l}\text { Porcentaje de mujeres empleadas con respecto } \\
\text { al total de trabajadores (\%) }\end{array}$ \\
\hline & & $\begin{array}{l}\text { Integración, diversidad e } \\
\text { igualdad de oportunidades. } \\
\text { Apoyo a las personas } \\
\text { vulnerables }\end{array}$ & $\begin{array}{l}\text { Trabajadores con } \\
\text { discapacidad } \\
\text { (DISCAP) }\end{array}$ & $\begin{array}{l}\text { Porcentaje de trabajadores con discapacidad } \\
\text { con respecto al total de trabajadores (\%) }\end{array}$ \\
\hline & Red profesional & $\begin{array}{l}\text { Inversión profesional en la } \\
\text { comunidad }\end{array}$ & $\begin{array}{l}\text { Gasto asociacionismo } \\
\text { (ASOCIA) }\end{array}$ & $\begin{array}{l}\text { Porcentaje de gasto destinado al asociacionismo } \\
\text { profesional con respecto al gasto total (\%) }\end{array}$ \\
\hline & $\begin{array}{l}\text { Compromiso } \\
\text { social }\end{array}$ & $\begin{array}{l}\text { Filantropia. Participación } \\
\text { en la comunidad. } \\
\text { Donaciones y otras } \\
\text { inversiones en la } \\
\text { comunidad }\end{array}$ & $\begin{array}{l}\text { Gasto donación y } \\
\text { filantropia } \\
\text { (DONA) }\end{array}$ & $\begin{array}{l}\text { Porcentaje de gasto destinado a donativos con } \\
\text { respecto al gasto total (\%) }\end{array}$ \\
\hline & $\begin{array}{l}\text { Profesionalización } \\
\text { interna }\end{array}$ & Formación & $\begin{array}{l}\text { Gasto formación } \\
\text { (FORMA) }\end{array}$ & $\begin{array}{l}\text { Porcentaje de gasto destinado a formación con } \\
\text { respecto al gasto total }(\%)\end{array}$ \\
\hline \multirow{6}{*}{ Económica } & \multirow{2}{*}{ Producción } & Capacidad productiva & $\begin{array}{l}\text { Valor de producción } \\
\text { (VALOR_PROD) }\end{array}$ & $\begin{array}{l}\text { Producción media por el precio del aceite de } \\
\text { oliva }(1000 €)\end{array}$ \\
\hline & & Productividad & $\begin{array}{l}\text { Productividad del trabajo } \\
\text { (PRODVIDAD) }\end{array}$ & 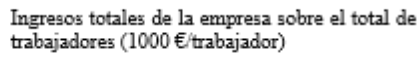 \\
\hline & \multirow{2}{*}{ Costes } & $\begin{array}{l}\text { Estructura financiera. } \\
\text { Liquidez. Fuentes externas }\end{array}$ & $\begin{array}{l}\text { Independencia financiera } \\
\text { (INDEPEN) }\end{array}$ & $\begin{array}{l}\text { Inversa del endeudamiento entendido como los } \\
\text { gastos financieros de la empresa con respecto a } \\
\text { sus gastos totales (adimensional) }\end{array}$ \\
\hline & & $\begin{array}{l}\text { Estructura financiera. } \\
\text { Liquidez. Autofinanciación }\end{array}$ & $\begin{array}{l}\text { Amortización del } \\
\text { immovilizado } \\
\text { (AMORTIZA) }\end{array}$ & $\begin{array}{l}\text { Porcentaje de la amortización sobre el total del } \\
\text { immovilizado (\%) }\end{array}$ \\
\hline & \multirow{2}{*}{ Ventas } & $\begin{array}{l}\text { Penetración en el mercado: } \\
\text { competitividad }\end{array}$ & $\begin{array}{l}\text { Cuota de mercado } \\
\text { (CUOTA) }\end{array}$ & $\begin{array}{l}\text { Porcentaje de las ventas de la empresa con } \\
\text { respecto al total de las ventas de aceite }(\%)\end{array}$ \\
\hline & & $\begin{array}{l}\text { Estrategia de producción: } \\
\text { creación de valor }\end{array}$ & $\begin{array}{l}\text { Economia circular } \\
\text { (CIRCULAR) }\end{array}$ & $\begin{array}{l}\text { Porcentaje de las ventas de subproductos del } \\
\text { olivar con respecto al total de las ventas (\%) }\end{array}$ \\
\hline \multirow{6}{*}{ Ambiental } & \multirow{2}{*}{ Agua } & $\begin{array}{l}\text { Prevención de la } \\
\text { contaminación del agua }\end{array}$ & $\begin{array}{l}\text { Control calidad del agua } \\
\text { (CONTROL_AGUA) }\end{array}$ & Control de la calidad del agua $(1=5 i, 0=n o)$ \\
\hline & & $\begin{array}{l}\text { Vertido de agua por } \\
\text { calidad y destino }\end{array}$ & $\begin{array}{l}\text { Destino agua de lavado } \\
\text { (CONTROL LAVADO) }\end{array}$ & $\begin{array}{l}\text { Destino del agua de lavado (l=vertido libre; } \\
\text { 2=balsa; } 3=\text { =depuradora) }\end{array}$ \\
\hline & Atmósfera & $\begin{array}{l}\text { Control de la } \\
\text { contaminación atmogférica }\end{array}$ & $\begin{array}{l}\text { Control emigiones gases } \\
\text { (CONTROL EMISION) }\end{array}$ & Control de las emisiones de gases $(1=5 \mathrm{i}, 0=n \mathrm{n})$ \\
\hline & Ruido & Control del nivel de ruido & $\begin{array}{l}\text { Control ruido } \\
\text { (CONTROL_RUIDO) }\end{array}$ & $\begin{array}{l}\text { Control del nivel de ruido a través de la } \\
\text { ubicación de la fabrica con respecto a la } \\
\text { población (l=dentro del municipio; } 2=\text { menos de } \\
2 \mathrm{~km} \text { del municipio; } 3=\text { más de } 2 \mathrm{~km} \text { del } \\
\text { municipio) }\end{array}$ \\
\hline & \multirow{2}{*}{$\begin{array}{l}\text { Materiales y } \\
\text { Energia }\end{array}$} & $\begin{array}{l}\text { Energia: energias } \\
\text { renovables }\end{array}$ & $\begin{array}{c}\text { Energía renovable y } \\
\text { Biomasa } \\
\text { (BIOMASA) }\end{array}$ & $\begin{array}{l}\text { Reciclaje y empleo de biomasa de origen propio } \\
(1=s i ; 0=n 0)\end{array}$ \\
\hline & & Materiales: reciclaje & $\begin{array}{l}\text { Reciclaje y uso } \\
\text { subproductos } \\
\text { (SUBPRODUCTO) }\end{array}$ & Ingresos por ventas de subproductos (1000€) \\
\hline
\end{tabular}


En la fase 2, se han construido índices sintéticos parciales de cada una de las dimensiones de sostenibilidad. Para ello, se ha hecho uso del análisis factorial (ACP) y del análisis envolvente de datos (DEA) (Zhou et al., 2018). Y en la fase 3, se ha realizado una agregación de los índices parciales para la construcción del índice global de sostenibilidad (GSI).

Finalmente, en la fase 4, con el objetivo de determinar qué factores inciden en cada dimensión de la sostenibilidad, se ha estimado un modelo de regresión truncada utilizando la técnica de bootstrap para evitar posibles sesgos en la estimación (Simar y Wilson, 2007). Las variables dependientes han sido, en cada caso, los índices parciales asociados a cada una de las dimensiones de la sostenibilidad, y el índice global, y las variables independientes las relacionadas con la dimensión de la almazara, con la infraestructura de la que dispone, y con el personal que realiza las funciones de gerencia.

\section{Resultados}

En el Cuadro 3 se incluye la descriptiva de los índices parciales y global de sostenibilidad estimados. Se observa cómo el índice parcial de sostenibilidad social (SPI_Soc) y el índice parcial de sostenibilidad económica (SPI_Eco) siguen una distribución similar, ambos con una media en torno a 0,60 y valores mínimos alrededor de 0,19-0,28. Los valores medios del índice parcial de sostenibilidad ambiental (SPI_Env) son superiores a los anteriores y se sitúan en torno a 0,76. El índice global de sostenibilidad (SGI) medio es de 0,65 , con un mínimo de 0,40 , sensiblemente superior a los índices parciales, y un máximo de 0,89 .

Cuadro 3. Estadísticos descriptivos de los índices parciales y global de la sostenibilidad

\begin{tabular}{l|cccc}
\hline \multirow{2}{*}{ Estadísticos } & \multicolumn{4}{|c}{ Índices de sostenibilidad } \\
\cline { 2 - 5 } & SPI_Soc & SPI_Eco & SPI_Env & SGI \\
\hline Media & 0,6 & 0,59 & 0,76 & 0,65 \\
Desviación estándar & 0,2 & 0,2 & 0,15 & 0,1 \\
Mínimo & 0,28 & 0,19 & 0,22 & 0,4 \\
Máximo & 1 & 1 & 1 & 0,89 \\
\hline
\end{tabular}

Los resultados obtenidos tras la aplicación de los cuatro modelos de regresión truncada bootstrap de los factores de sostenibilidad se presentan en el Cuadro 4 y muestran diferentes relaciones significativas. Así, se puede verificar que:

Cuadro 4. Regresión truncada bootstrap para los índices de sostenibilidad ${ }^{1}$

\begin{tabular}{|c|c|c|c|c|}
\hline \multirow{2}{*}{ Índices de sostenibilidad } & \multirow{2}{*}{$\begin{array}{c}\text { Coeficiente } \\
\text { observado }\end{array}$} & \multirow{2}{*}{ Boostrap Std. Err. } & \multicolumn{2}{|c|}{ [95\% Conf. Interval] } \\
\hline & & & Lower & Upper \\
\hline \multicolumn{5}{|l|}{ SPI_Soc } \\
\hline Constante & 0,6669 & 0,1297 & 0,4035 & 0,9305 \\
\hline Tamaño & $-0,0085^{* * *}$ & 0,0024 & $-0,0133$ & $-0,0041$ \\
\hline Trabajo & $0,0026^{* * *}$ & 0,0007 & 0,0013 & 0,0042 \\
\hline Lineas & $0,0305^{* \bullet}$ & 0,0144 & 0,0025 & 0,0605 \\
\hline Envasadora propia & $0,0860^{*}$ & 0,0477 & $-0,0055$ & 0,1784 \\
\hline Certificados & $0.1050^{*}$ & 0,0554 & 0,0011 & 0.2202 \\
\hline Sexo & $-0,1891^{\bullet+}$ & 0,0565 & $-0,3011$ & $-0,0850$ \\
\hline Sigma & 0,1470 & 0,0127 & 0,1096 & 0,1589 \\
\hline \multicolumn{5}{|l|}{ SPI_Eco } \\
\hline Constante & 0,4545 & 0,1108 & 0,2397 & 0,6882 \\
\hline Tamaño & $0,0082^{* * *}$ & 0,0022 & 0,0040 & 0,0125 \\
\hline Trabajo & $-0,0022^{\bullet+}$ & 0,0010 & $-0,0042$ & $-0,0002$ \\
\hline Estudios básicos & $-0,1091^{*}$ & 0,0570 & $-0,2196$ & 0,0015 \\
\hline Formación gerencia & $0,0953^{* *}$ & 0,0432 & 0,0114 & 0,1826 \\
\hline Sigma & 0,1341 & 0,0112 & 0,0998 & 0,1441 \\
\hline \multicolumn{5}{|l|}{ SPI_Env } \\
\hline Constante & 0,6748 & 0,1121 & 0,4581 & 0,8837 \\
\hline Tamafio & $0,0039^{*}$ & 0,0021 & $-0,0002$ & 0,0082 \\
\hline Sexo & $0,0926^{* *}$ & 0,0456 & 0,0050 & 0,1828 \\
\hline Estudios universitarios & $0,0976^{* *}$ & 0,0461 & 0,0007 & 0,1840 \\
\hline Formación gerencia & $-0,0700^{*}$ & 0,0419 & $-0,1506$ & 0,0147 \\
\hline Sigma & 0,1208 & 0,0113 & 0,0863 & 0,1309 \\
\hline \multicolumn{5}{|l|}{ SGI } \\
\hline Constante & 0,5890 & 0,0669 & 0,4507 & 0,7124 \\
\hline Certificados & $0,0610^{8 * x}$ & 0,0283 & 0,0085 & 0,1179 \\
\hline Sigma & 0,0833 & 0,0069 & 0,0630 & 0,0898 \\
\hline
\end{tabular}

${ }^{1}$ Número de observaciones $=81 ;$ número de replicaciones $=1.000 .{ }^{*} \mathrm{p}<0,1 ;{ }^{* *} \mathrm{p}<0,05 ;{ }^{* * *} \mathrm{p}<0,001$. Se omiten las relaciones no significativas. Categoría omitida es estudios intermedios. 
- la sostenibilidad social disminuye a medida que aumenta el tamaño de las cooperativas. En cambio, aumenta conforme se contrata más personal y de género femenino, y cuanto mejor es la infraestructura de la cooperativaen lo que respecta al número de líneas de la almazara, disponibilidad de envasadora propia y certificaciones de calidad.

- la sostenibilidad económica es mayor conforme aumenta el tamaño de la almaraza y disminuye el número de trabajadores, y cuanto mayor es tanto el nivel de estudios del responsable de la administración como su formación en el ámbito de la gestión.

- la sostenibilidad ambiental es mayor conforme aumenta el tamaño de la almazara y las funciones de gerencia las realizan hombres con nivel de estudios universitarios y una formación más centrada en dar respuestas a los problemas ecológicos que a los de gestión.

- la sostenibilidad global mejora si la almazara cuenta con certificaciones de calidad.

\section{Conclusiones}

En esta investigación se ha avanzado en el diseño de una propuesta metodológica que permite abordar tanto la medición de la sostenibilidad de las almazaras, como los factores que sobre ella inciden. En base a los resultados obtenidos se pueden realizar las siguientes consideraciones conclusivas: i) la dimensión de la almazara y el incremento del tamaño empresarial se erigen como factores fundamentales para avanzar hacia procesos más sostenibles; ii) en un contexto como en el que se desenvuelve el sector del aceite de oliva, caracterizado por una fuerte internacionalización de la producción y unos consumidores cada vez más informados y sensibilizados por los valores nutricionales y de salud de los alimentos, la apuesta por la calidad se convierte no sólo en una oportunidad, sino en una necesidad; iii) la formación y profesionalización de los responsables de la gestión de las almazaras es un elemento fundamental para la sostenibilidad empresarial.

\section{Bibliografía}

Ahmad, S., Wong, K. Y. y Zaman, B. (2019). “A Comprehensive and Integrated Stochastic-Fuzzy Method for Sustainability Assessment in the Malaysian Food Manufacturing Industry”. Sustainability, 11(4).

Bellantuono, N., Pontrandolfo, P. y Scozzi, B. (2018). "Guiding materiality analysis for sustainability reporting: The case of agri-food sector". International Journal of Technology, Policy and Management, 18(4), 336-359.

Junta de Andalucía. (2020). Caracterización del sector agrario y pesquero de Andalucía. Consejería de agricultura, ganadería, pesca y desarrollo sostenible. Junta de Andalucía.

Notarnicola, B., Tassielli, G., Renzulli, P. A., Castellani, V. y Sala, S. (2017). "Environmental impacts of food consumption in Europe". Journal of Cleaner Production, 140, 753-765.

Roig, A., Cayuela, M. L. y Sánchez-Monedero, M. A. (2006). “An overview on olive mill wastes and their valorisation methods". Waste Management, 26(9), 960-969.

Salvado, M. F., Azevedo, S. G., Matias, J. C. O. y Ferreira, L. M. (2015). "Proposal of a sustainability index for the automotive industry". Sustainability, 7(2), 2113-2144.

Simar, L. y Wilson, P. W. (2007). "Estimation and inference in two-stage, semi-parametric models of production processes". Journal of Econometrics, 136(1), 31-64.

Zhou, H., Yang, Y., Chen, Y. y Zhu, J. (2018). "Data envelopment analysis application in sustainability: The origins, development and future directions". European Journal of Operational Research, 264(1), 116.

Agradecimientos: Esta investigación ha sido financiada por el Grupo Operativo BIOECOLIVA (GOP3IMA-16-0004) cofinanciado por la Unión Europea a través del Fondo Europeo Agrario de Desarrollo Rural (FEADER) y la Junta de Andalucía a través de la Consejería de Agricultura, Ganadería, Pesca y Desarrollo Sostenible. 